\title{
海洋生物中具有生理活性的多羟基甾醇
}

\author{
林啟福 崔建国* 甘春芳刘亮姚秋翠 黄燕敏* \\ (广西师范学院化学与生命科学学院 南宁 530001)
}

\begin{abstract}
摘要 多羟基甾醇是一类具有显著生理活性的天然化合物, 广泛地存在于海洋生物当中. 根据甾体中所含羟基的数目 进行分类，综述了近几年来从海洋生物中分离得到的具有生理活性的多羟基甾醇的研究进展.
\end{abstract}

关键词 海洋生物; 生理活性; 多羟基甾醇

\section{Polyhydroxysterols with Bioactivities from Marine Organisms}

\author{
Lin, Qifu Cui, Jianguo* Gan, Chunfang Liu, Liang Yao, Qiucui Huang, Yanmin* \\ (College of Chemistry and Life Science, Guangxi Teachers Education University, Nanning 530001)
}

\begin{abstract}
Polyhydroxysterols are a kind of natural compounds with significant physiological activities, widely exist in marine organisms. Recent researches on polyhydroxysterols with bioactivities isolated from marine organisms are reviewed according to the number of hydroxyl group on sterols.

Keywords marine organism; biological activity; polyhydroxysterol
\end{abstract}

海洋具有丰富的生态资源和特殊的生态环境, 生物 物种之间的生存竞争十分激烈, 为了能够在严酷的环境 下进化生存, 迫使很多海洋生物在生命过程中代谢产生 一些结构特殊、生物活性显著的化学物质, 即次生代谢 产物. 现代药理学研究表明, 很多海洋次生代谢产物对 人类多种疾病有着很好的治疗作用, 因而引起化学家、 生物学家、药理学家的极大兴趣 ${ }^{11}$. 多羟基甾醇就是一 类具有较好生理和药理活性的化合物, 一直引起海洋化 学家和药物学家的研究兴趣.

近年来, 国内外对于从海绵、海藻、棘皮动物等分 离得到的多羟基甾醇类化合物已有较多的研究报道. 这 里, 我们根据甾体所含的羟基个数进行分类, 综述近几 年来从海洋生物中分离得到的具有生理活性的多羟基 甾醇的研究进展.

\section{1 单羟基甾醇化合物}

Wang 等 ${ }^{[2]}$ 从海洋真菌 Rhizopus $s p$. 的乙酸乙酯提 取液中分离得到八个含一个羟基的甾体化合物 $\mathbf{1} \sim \mathbf{8}$, 并对这些化合物进行了体外抗肿瘤活性测试. 结果表明 它们对于 P388, A549, HL-60, BEL-7420 这 4 种癌细胞
都具有较好的抑制作用，其中对这四种癌细胞抑制效果 最好的分别是: 化合物 2 (P388 细胞株, $\mathrm{IC}_{50}$ 值: 2.0 $\mu \mathrm{mol} / \mathrm{L}$ ), 化合物 4 (A549 细胞株, $\mathrm{IC}_{50}$ 值: $6.6 \mu \mathrm{mol} / \mathrm{L}$ ), 化合物 8 (HL-60 细胞株, $\mathrm{IC}_{50}$ 值: $2.7 \mu \mathrm{mol} / \mathrm{L}$ ), 化合物 $\mathbf{1}$ (BEL-7420 细胞株, $\mathrm{IC}_{50}$ 值: $5.9 \mu \mathrm{mol} / \mathrm{L}$ ). 而且通过分析 发现这些化合物对 P388, HL-60 的抑制效果普遍要比 A549, BEL-7420 好. 对比这些化合物的结构, 我们发现 这些化合物在 3-位上都存在着一个 $\beta$-羟基, 同时, 所有 化合物都具有类似于豆甾醇类型的支链结构.

Nguyen 等 ${ }^{[3]}$ 从越南海绵 Ianthella $s p$. 中分离出 3 个 化合物 9 11, 通过体外细胞毒活性实验发现这 3 个化 合物对于 A549, HL-60, MCF-7, SK-OV-3, U937 五种肿 瘤细胞都具有较好的抑制活性, 测得的 $\mathrm{IC}_{50}$ 值在 8.4 $22.6 \mu \mathrm{mol} / \mathrm{L}$ 之间, 它们对 A549 肿瘤细胞的抑制效果最 好, 其 $\mathrm{IC}_{50}$ 值分别为: $8.4,9.8$ 和 $11.5 \mu \mathrm{mol} / \mathrm{L}$; 而对于 HL-60 肿瘤细胞则表现出弱的抑制活性, 这些数据表明 这三个化合物具有治疗白血病的潜在能力. 对比这三个 化合物的结构, 我们可以发现在这些化合物支链的 25位和 26-位都存在着一个环丙烷结构.

Marino 等 $^{[4]}$ 从马来塔岛西海岸的海绵 Theonella

\footnotetext{
*E-mail: cuijg1954@126.com; huangyanmin828@163.com

Received July 7, 2012; revised August 15, 2012; published online August 22, 2012.

Project supported by the Natural Science Foundation of Guangxi Province (Nos. 2010GXNSFD013019, 2010GXNSFA013052).

广西自然科学基金(Nos. 2010GXNSFD013019, 2010GXNSFA013052)资助项目.
} 


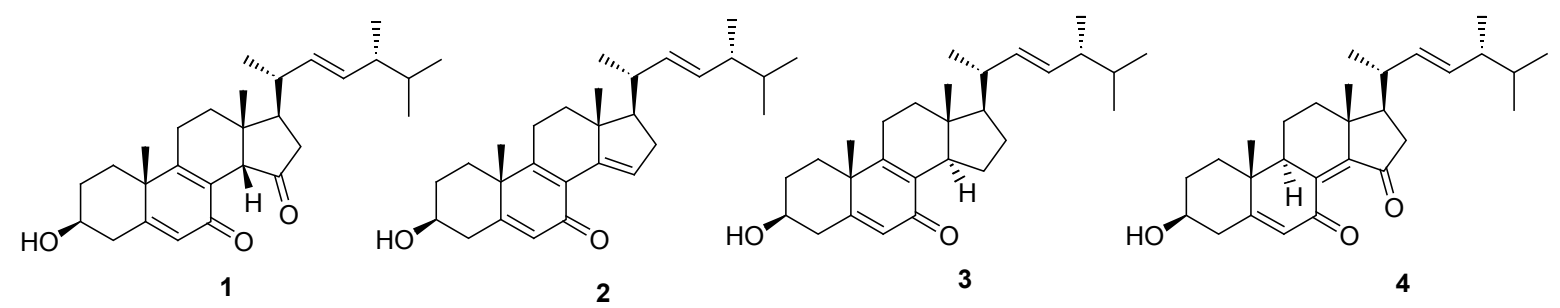<smiles>[R7]CC(C)(C)/C=C(/C)CC(C)C</smiles>

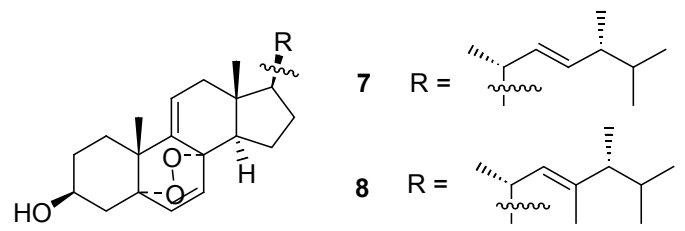<smiles>CC(CCC(C)C1CCC2C3CC(=O)C=C4CC(=O)CCC4(C)C3CCC12C)C1CC1</smiles>

9<smiles>CC(CCC(C)C1CCC2C3CC4CC(O)CCC4(C)C3CCC12C)C1CC1</smiles>

10

11

swinhoei 中分离得到化合物 $\mathbf{1 2} \sim \mathbf{1 7}$, 通过转录分析研究 发现浓度为 $50 \mu \mathrm{mol} / \mathrm{L}$ 的化合物 12, 15 和 17 在 $10 \mu \mathrm{mol} / \mathrm{L}$ 我去氧胆酸 CDCA 的诱导下, 都表现出一定的抑制胆汁 酸受体(FXR)转录的活性. 同时, 研究发现化合物 13, $14,15,17$ 均表现出很好的诱导㝋烷 X受体(PXR)转录的 活性, 其中化合物 17 诱导 PXR 转录的活性与药物利福 昔明相当. 这些研究成果为发展兼具强大免疫调节活性 和调控胆汁酸调控基因表达活性的药物提供了理论基 础. 此类化合物的甾核结构中都存在一个 4-位亚甲基, 而具有这种甾核结构的甾体化合物在天然甾体中是比 较少见的.

Shin 等 ${ }^{[5]}$ 从软珊瑚 Dendronephthya gigantea 中分离 出五个单羟基化合物 $\mathbf{1 8} \sim \mathbf{2 2}$, 研究发现这五个化合物都 具有一定的抑制胆汁酸受体(FXR)的活性, 测得其 $\mathrm{IC}_{50}$ 值分别为: 14, 15, 100, 22 和 $61 \mu \mathrm{mol} / \mathrm{L}$.

\section{2 二羟基甾醇化合物}

Sheu 小组 ${ }^{[6-8]}$ 从台湾软珊瑚 Sinularia $s p$. 和台湾八 放珊瑚 Dendronephthya griffini 中分离得到 8 个含有 2 个 美基的甾体化合物 $\mathbf{2 3} \sim \mathbf{3 0}$, 同时对这些化合物进行了体 外抗炎活性测试, 结果表明化合物 23, 24 和 29 在浓度为 $10 \mu \mathrm{mol} / \mathrm{L}$ 时，对脂多糖诱导下 RAW264.7 巨噬细胞的 促炎 COX-2 蛋白的累积具有很好的抑制作用. 化合物 23,24 和 25,26 的结构相比, 唯一的差别是化合物 25 与 26 在甾核的 9,11-位存在一个双键，而化合物 23, 24 在 这个位置没有双键. 从这里可以看出, 甾核结构的微小 变化都会对甾体母体化合物的生理活性产生较大的改 变. 化合物 27, 28, 29 和 30 在浓度为 $10 \mu \mathrm{mol} / \mathrm{L}$ 时, 对脂 多糖诱导下 RAW264.7 巨噬细胞的促炎 iNOS 蛋白的 累积具有很好的抑制作用.

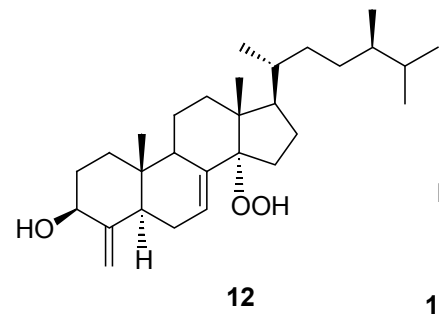

$14 \mathrm{R}=$<smiles>[R]C1CC=C2C3=CCC4C(=C)C(O)CCC4(C)C3CCC21C</smiles><smiles>CC(C)C(C)CC[C@H](C)C(C)C</smiles>
$16 R=$<smiles>C=C(CCC(C)C)C(C)C</smiles>

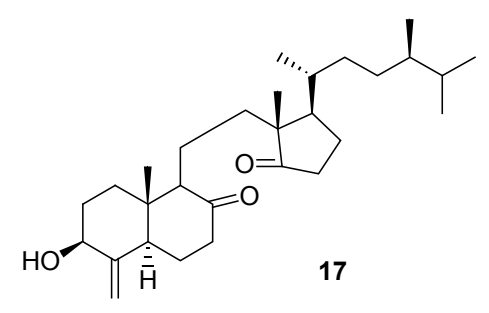



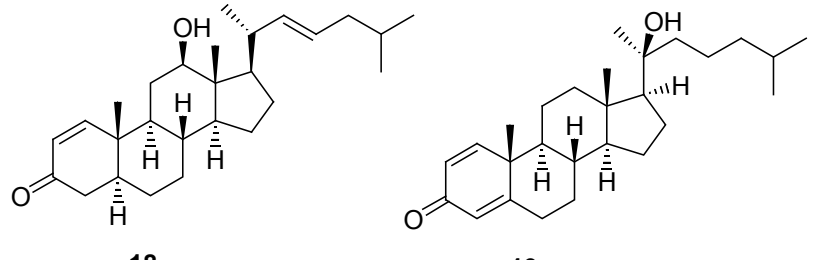

19

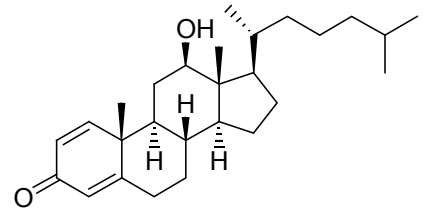

20<smiles>C=C(CC[C@](C)(O)[C@H]1CCC2C3CCC4=CC(=O)C=C[C@]4(C)C3CC[C@]21C)C(C)C</smiles>

21

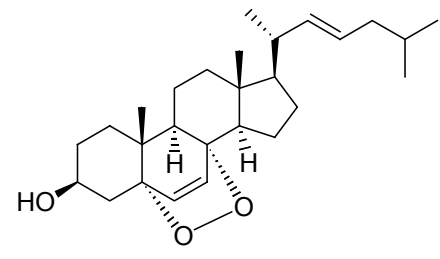<smiles>[R4]C[C@H](C)[C@@H](C)CCC(=C)C(C)C</smiles>

22

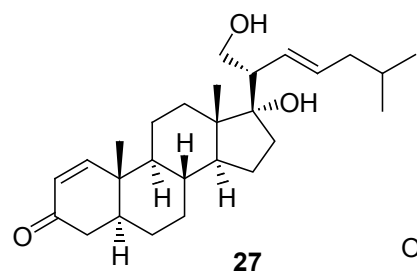

27

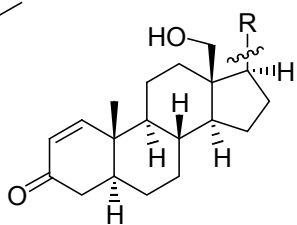

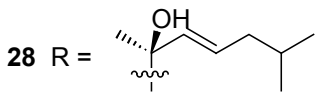

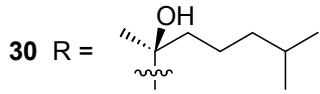

Wang 等 ${ }^{[9]}$ 从台湾软珊瑚 Stereonephthya crystalliana 的二氯甲烷提取液中分离得到一个二羟基甾醇化 合物 31, 并对 31 进行了体外抗肿瘤活性测试, 结果表 明该化合物对于人结肠癌细胞 HT-29 和小鼠白血病细 胞 P-388 具有较好的活性, 其半数有效量( $\mathrm{ED}_{50}$ 值)分别 为 7.5 和 $3.5 \mu \mathrm{g} / \mathrm{mL}$.
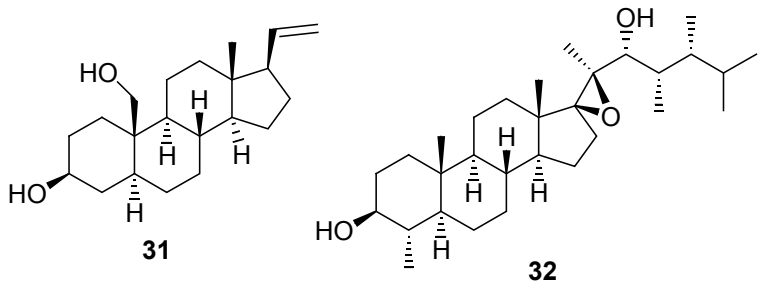

Tran 等 ${ }^{[10]}$ 从越南软珊瑚 Lobophytum laevigatum 的 甲醇提取液中分离得到新化合物 32 , 对其进行体外抑 制肿瘤细胞生长增殖活性测试结果表明, 其对 A549,
HCT-116, HL-60 三种癌细胞都具有较好的抑制作用, $\mathrm{IC}_{50}$ 值分别为: $(4.5 \pm 0.5),(3.2 \pm 0.9),(5.6 \pm 0.4) \mu \mathrm{mol} / \mathrm{L}$. 而且通过进一步的实验研究发现该化合物主要是通过 诱导细胞调亡的方式来抑制细胞的增长. 从结构上看, 在该化合物的 17,20-位存在着一个环氧结构.

Simmons 等 ${ }^{[11]}$ 从海绵源性细菌 Actinomadura $s p$. SBMs009 中分离得到化合物 33 35, 通过实验研究发 现：化合物 33 对于小鼠成纤维细胞 L929 有中等强度 的抑制活性，其 $\mathrm{IC}_{50}$ 值约为 $30 \mu \mathrm{mol} / \mathrm{L}$, 而且还发现这三 个化合物都有抑制糖皮质激素受体(GR)易位的活性，其 中化合物 $\mathbf{3 3}$ 的抑制效果最好; 此外，化合物 $\mathbf{3 5}$ 还有较 好的抑制 NF- $\mathrm{kB}$ 核易位的活性，其 $\mathrm{IC}_{50}$ 值为 $71 \mu \mathrm{mol} / \mathrm{L}$.

Zhou 等 ${ }^{[12]}$ 从中国南海海绵 Xestospongia testudinaria 中分离得到化合物 36 39, 研究发现这四个化合 物对于卤虫 Artemia salina 幼虫有很好的毒活性, 其 $\mathrm{LD}_{50}$ 值(半数致死浓度)分别为: $0.56,5.89,12.45$ 和 0.63 

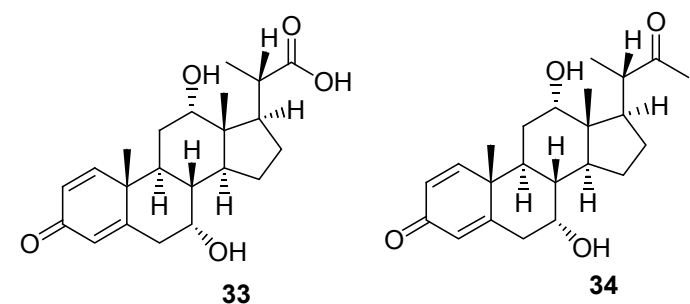<smiles>C[C@H](CCC(=O)O)[C@H]1CC[C@H]2[C@@H]3[C@H](C[C@H](O)[C@]21C)[C@@]1(C)C=CC(=O)C=C1C[C@H]3O</smiles>

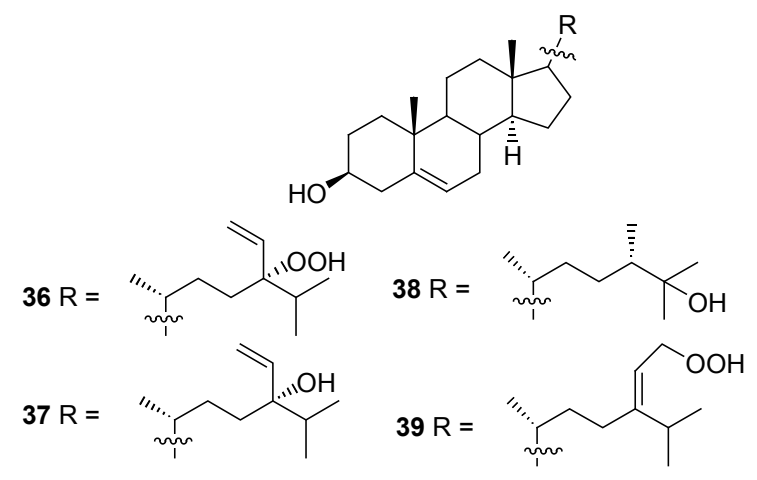

$\mu \mathrm{mol} / \mathrm{L}$. 同时还发现化合物 36 和 39 表现出弱的抑制 $\mathrm{AChE}$ (乙酰胆碱酯酶) 活性, 其 $\mathrm{IC}_{50}$ 值分别为 11.45 和 $14.51 \mu \mathrm{mol} / \mathrm{L}$. 化合物 36, 39 从结构上来看都存在着一 个过氧氢基.

\section{3 三羟基甾醇化合物}

三羟基甾醇化合物是海洋甾醇中报道较多的一类, 它们往往具有多种生理活性, 如抗肿瘤、抗症疾、抗炎、 抗菌、保肝、抑制蛋白酶等活性.

\section{1 抗肿瘤活性}

Ahmed 等 ${ }^{[13]}$ 从软珊瑚 Sinularia grandilobata 的乙醇 提取液中分离得到四个新的三差基甾醇化合物 $40 \sim \mathbf{4 3}$, 体外抑制肿瘤细胞生长增殖活性测试表明：化合物 40 对人乳腺癌细胞 MDA-MB-231 有较好的抑制作用, 其 $\mathrm{IC}_{50}$ 值为 $9.2 \mu \mathrm{g} / \mathrm{mL}$; 化合物 $\mathbf{4 1}$ 对人肝癌细胞 Hep $\mathrm{G} 2$ 和人肺癌细胞 A549 有较好的抑制作用, 其 $\mathrm{IC}_{50}$ 值分别 为 6.8 和 $16.9 \mu \mathrm{g} / \mathrm{mL}$; 化合物 42 则对人乳腺癌细胞 $\mathrm{MCF}-7$ 有较好的抑制作用, $\mathrm{IC}_{50}$ 值为 $18.9 \mu \mathrm{g} / \mathrm{mL}$; 化合 物 43 对这几种癌细胞基本没有活性. 化合物 43 与 42 的结构相比较, $\mathbf{4 3}$ 在支链的 22,23-位存在一个碳碳双键, 这个双键与支链中的 24,28-位的双键形成了共轭结构, 正是受这个共轭双键的影响，使得其细胞毒活性消失.

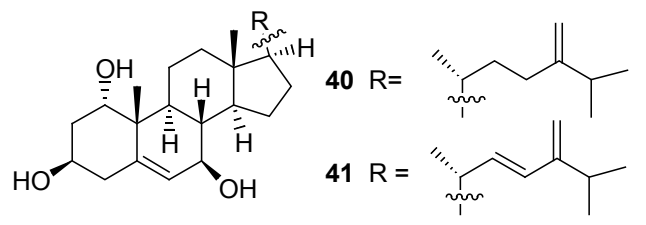

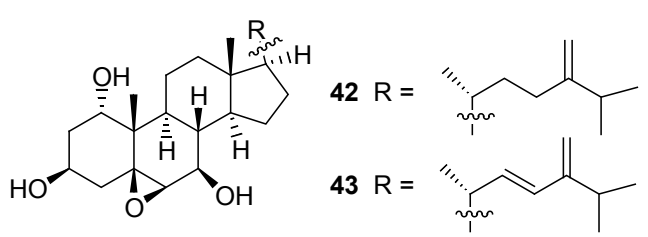

Duh 等 ${ }^{[14]}$ 从台湾软珊瑚 Nephthea erecta 的丙酮提取 液中分离得到化合物 $44 \sim \mathbf{4 7}$, 通过细胞毒活性实验发 现：化合物 45, 47 对于白血病细胞 P388 和人结肠癌细 胞 HT-29 有较好的活性，其 $\mathrm{ED}_{50}$ 值分别为 3.8, 3.6 和 4.7, $4.3 \mu \mathrm{g} / \mathrm{mL}$, 而化合物 46 对这两种细胞没有毒性. 从结 构上来看, 化合物 46 与 45,47 相比较, 也只是在支链上 有微小的差别, 从而使其对这两种肿瘤细胞失去了活 性.<smiles>C=C(CC[C@H](C)C1CC[C@H]2[C@H]3C(O)C=C4C[C@@H](O)CCC4(CO)[C@H]3CC[C@]12C)C(C)C</smiles>

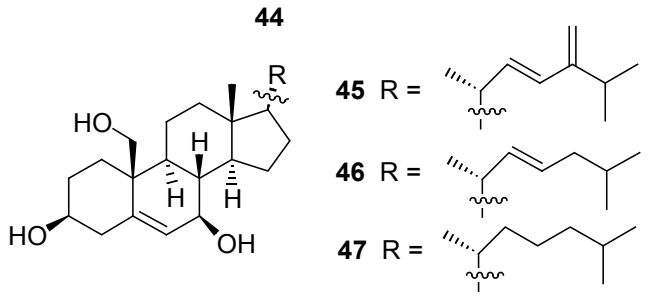

\section{2 抗疮疾活性}

Rao 等 ${ }^{[15]}$ 从印度孟加拉湾西部的马纳尔湾收集到 的海绵 Callyspongia fibrosa 中分离得到化合物 48, 通 过研究发现其对于恶性虐原虫(Plasmodium falciparum) 有中等强度的活性. 而且与氯喹相反, 其对恶性虐原虫 氯喹抗性株的活性要比对氯喹敏感株的活性要好.

\section{3 保肝活性}

Sepe 等 ${ }^{[16]}$ 从所罗门群岛的海绵 Theonella swinhoei 中分离得到化合物 49, 通过药理研究发现其具有调控 胆汁酸调控基因表达的活性，表现出胆汁酸受体(FXR) 调节剂和孕烷 X 受体(PXR)激动剂的作用, 具有治疗肝 脏疾病的效用. 此化合物同样在其甾核的 4-位存在一个 亚甲基取代. 
<smiles>CCCC1(C)CCC2C3CC(O)[C@@]4(O)C[C@@H](O)CCC4(C)C3CCC21C</smiles>

48
49

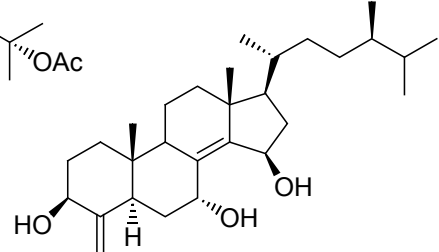

\section{4 抗炎活性}

Huang 等 ${ }^{[17]}$ 从台湾软珊瑚 Nephthea chabroli 的丙 酮提取液中分离出六个含三个羟基的甾体化合物 50 55, 并使用脂多糖诱导对 RAW264.7 巨噬细胞进行体外 抗炎活性测试，结果表明：化合物 $\mathbf{5 1 , 5 3}$ 和 54 在浓度为 $10 \mu \mathrm{mol} / \mathrm{L}$ 时, 能够显著地减少 $\mathrm{iNOS}$ 的量到 $(0 \pm 0) \%$, $(43.1 \pm 7.9) \%$ 和 $(76.9 \pm 9.4) \%$, 同时减少 COX-2 的量到 $(63.9 \pm 10.9) \% ，(57.4 \pm 5.1) \%$ 和 $(72.0 \pm 10.5) \%$. 化合物 50 和 55 只能够有效地抑制 iNOS 蛋白的表达 [(10.6土 $0.5) \%,(32.8 \pm 6.8) \%$ ], 而不能抑制 COX-2 蛋白的表达; 化合物 52 则不能抑制这两种蛋白的表达. 在同样的实 验条件下, $10 \mu \mathrm{mol} / \mathrm{L}$ 的 CAPE(咖啡酸苯乙酯)能够抑制 iNOS 和 COX-2 的量到 $(1.5 \pm 2.1) \%$ 和 $(70.2 \pm 11.5) \%$.

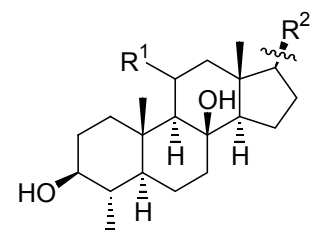

$$
\begin{array}{ll}
50 \mathrm{R}^{1}=\beta-\mathrm{OH}, \mathrm{R}^{2}=\mathrm{S}_{1} & 53 \mathrm{R}^{1}=\beta-\mathrm{OH}, \mathrm{R}^{2}=\mathrm{S}_{4} \\
51 \mathrm{R}^{1}=\beta-\mathrm{OH}, \mathrm{R}^{2}=\mathrm{S}_{2} & 54 \mathrm{R}^{1}=\beta-\mathrm{OH}, \mathrm{R}^{2}=\mathrm{S}_{5} \\
52 \mathrm{R}^{1}=\beta-\mathrm{OH}, \mathrm{R}^{2}=\mathrm{S}_{3} & 55 \mathrm{R}^{1}=\mathrm{H}, \mathrm{R}^{2}=\mathrm{S}_{6}
\end{array}
$$<smiles>C=C(C(=O)[C@@H](C)CC(=O)C(C)C(=C)/C=C\C(C)C)C(C)C</smiles><smiles>C=C(/C=C/[C@H](C=S)N(C)C)C(C)C</smiles>

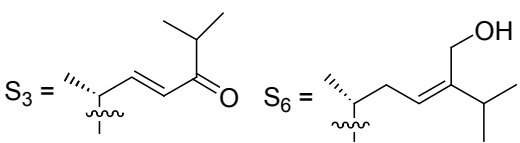

\section{5 抗菌活性}

Sauleau 等 ${ }^{[18]}$ 从红海海绵 Lamellodysidea herbacea 中分离得到化合物 56, 57, 通过抗菌试验发现这两个化 合物在溶度为 $100 \mu \mathrm{g} / \mathrm{disc}$ 时, 对于金黄色葡萄球菌 $S$. aureus 和大肠杆菌 E. coli 均没有抗菌活性, 而对于热带 念珠菌 Candida tropicalis 则表现出一定的抗菌作用(溶 度为 $10 \mu \mathrm{g} / \mathrm{disc}$ 时, 对于直径为 11 和 $13 \mathrm{~mm}$ 的菌株有很 好的抗菌效果). 此两个化合物都属于 3,5,6-三羟基甾醇.

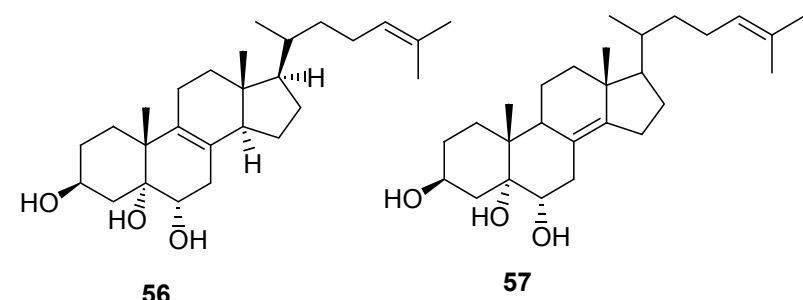

\section{6 抑制天冬氨酸蛋白酶(BACE1)的活性}

Dai 等 ${ }^{[19]}$ 从海绵 Topsentia $s p$. 中分离得到化合物 $58 \sim 61$, 通过实验研究发现: 化合物 61 具有较好的抑制 天冬氨酸蛋白酶(BACE1)的活性 $\left(\mathrm{IC}_{50}\right.$ 值: $\left.1.2 \mu \mathrm{mol} / \mathrm{L}\right)$, 而化合物 $58 \sim 60$ 则不具有这种抑制活性，进一步分析 结果表明化合物 61 之所以有较好活性与其存在着硫酸 酯钠基有关. 而且从化合物 58 和 $\mathbf{6 1}$ 的结构对比也可以 发现，化合物 61 是化合物 $\mathbf{5 8}$ 的三个羟基形成硫酸酯钠 盐后的产物.
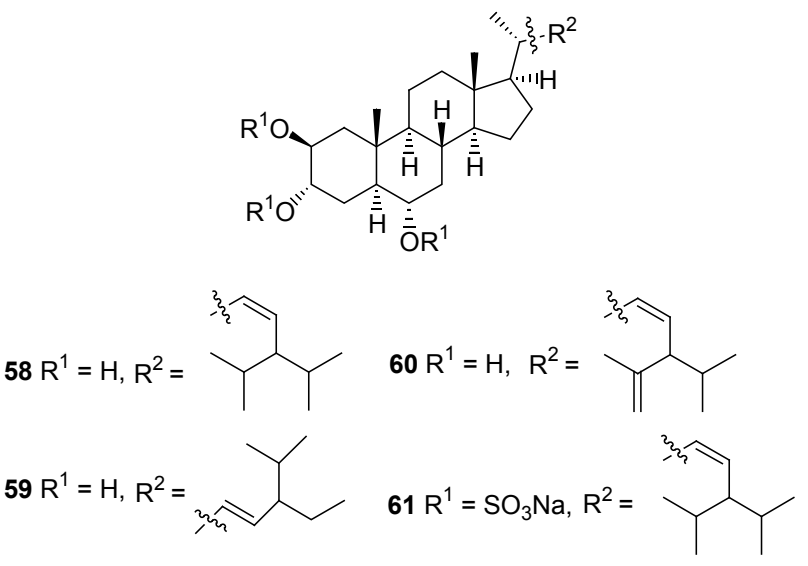

\section{4 四羟基甾醇类化合物}

Mitome 等 ${ }^{[20]}$ 从海绵 Stylissa sp. 中分离得到一个四 差基甾醇化合物 62, 并进行了体外细胞毒性实验, 发现 其对海拉宫颈癌细胞有一定的抑制作用，其 $\mathrm{IC}_{50}$ 值为 $27.2 \mu \mathrm{mol} / \mathrm{L}$.

张福勇等 ${ }^{[21]}$ 从中国南海砶洲岛软珊瑚 Chromonephthea $s p$. 中分离鉴定出两个四羟基甾醇化合物 $\mathbf{6 3}, \mathbf{6 4}$, 体外抗肿瘤活性测试结果表明，这两个甾醇对 BEL7402, HeLa 和 MCF 肿瘤细胞具有一定的抑制作用. 其 中化合物 63, 64 对这三种肿瘤细胞株的 $\mathrm{IC}_{50}$ 值分别为 $51.68,66.51,12.88 \mu \mathrm{g} / \mathrm{mL}$ 和 $4.97,19.41,19.01 \mu \mathrm{g} / \mathrm{mL}$.

廖小建等 ${ }^{[22]}$ 从中国南海䂵洲岛软珊瑚 Dendronephthya $s p$. 中分离得到两个四羟基甾醇化合物 $\mathbf{6 5}, \mathbf{6 6}$, 其中 化合物 65 是未见报道的新化合物，化合物 66 也是首次 从该属珊瑚中获得，体外细胞毒活性试验表明：化合物 65 对肿瘤细胞 BEL-7402, MCG, MCF, LOVO 和 HepG2 具有一定的抑制作用, $\mathrm{IC}_{50}$ 值分别为 $32.2,20.5,2.0,5.5$ 
和 $18.6 \mu \mathrm{g} / \mathrm{mL}$; 而化合物 66 对于肿瘤细胞 MCG 和 LOVO 具有中等强度的抑制作用, $\mathrm{IC}_{50}$ 值分别为 22.0 和 $13.8 \mu \mathrm{g} / \mathrm{mL}$.

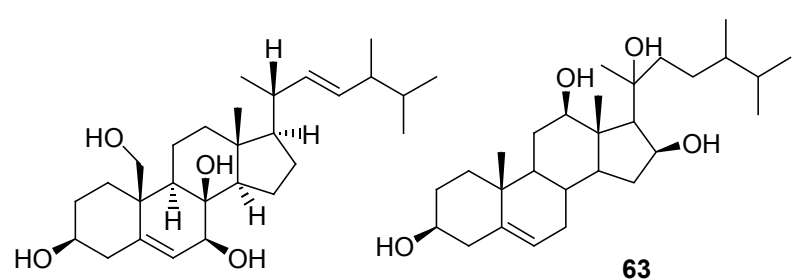

63<smiles>C=C(C(C)C)C(C)C1CCC2C3C(CCC12C)C1(C)CC[C@@H](O)C[C@]1(O)[C@H](O)C(O)C3(C)C</smiles>

64

65
70 72, 通过体外抗炎活性试验发现化合物 70, 71 表现 出一定的抗炎活性.

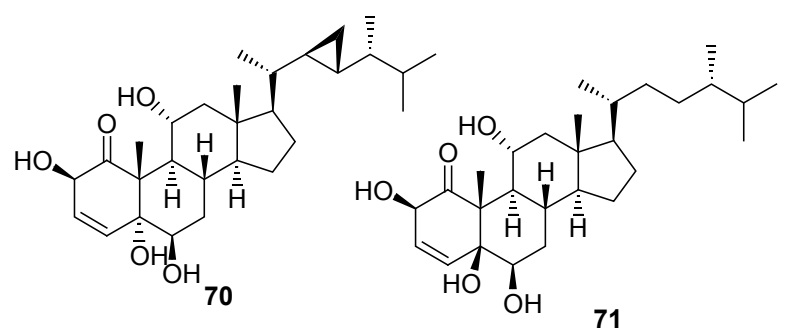

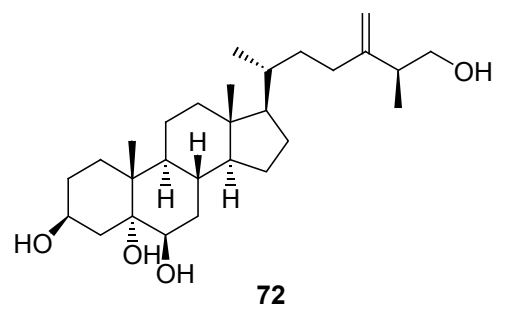

\section{5 五羟基甾醇化合物及其甾体皇苷}

Wang 等 ${ }^{[26]}$ 从海星 Certonardoa semiregularis 的甲 醇提取液中分离得到两个新的硫酸酯化甾体皇苷化合 物 73, 74, 同时进行了体外抗肿瘤活性测试, 结果发现 化合物 73 对肿瘤细胞株 SK-OV-3, SK-MEL-2, XF498 和 HCT15 具有一定的活性, 其中化合物 73 对 SK-MEL2 的活性最好, $\mathrm{ED}_{50}$ 为 $2.67 \mu \mathrm{g} / \mathrm{mL}$; 化合物 74 的 $\mathrm{ED}_{50}$ 值 却大于 $30 \mu \mathrm{g} / \mathrm{mL}$.
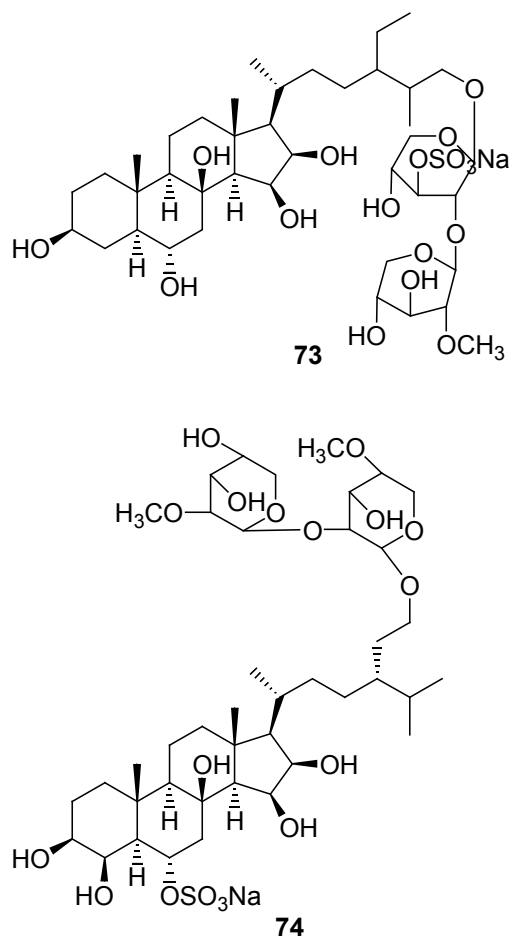

$\mathrm{Ma}$ 等 ${ }^{[27]}$ 从海星 Anthenea chinensis 的乙醇提取液 中分离鉴定出十种新的多羟基甾体皇苷类化合物 75 甲烷提取液中分离出三个含有四个羟基的甾体化合物 
84, 并进行了体外抑制肿瘤细胞活性测试, 结果表明: 化合物 78, 79, 81 和 82 的混合物以及化合物 83 和 84 的 混合物对于肿瘤细胞 K-562 和 BEL-7402 均具有很好的 抑制作用, 其 $\mathrm{IC}_{50}$ 值分别为: $(6.1 \pm 1.4),(4.6 \pm 1.1)$, $(2.8 \pm 0.9),(0.6 \pm 0.1) \mu \mathrm{mol} / \mathrm{L}$ 和 $(8.3 \pm 1.9),(5.5 \pm 1.0)$, $(4.9 \pm 1.5),(2.7 \pm 0.5) \mu \mathrm{mol} / \mathrm{L}$. 化合物 83,84 的混合物对 于 $\mathrm{U} 87 \mathrm{M}$ 肿瘤细胞也有较好的活性, $\mathrm{IC}_{50}$ 值为 $(3.6 \pm 0.9)$ $\mu \mathrm{mol} / \mathrm{L}$. 除此之外, 化合物 83,84 的混合物还具有促进 微管蛋白聚合的活性.
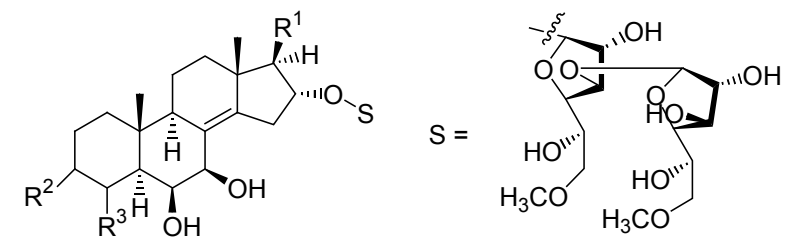

$75 \mathrm{R}^{1}=\mathrm{A}_{1}, \mathrm{R}^{2}=\beta-\mathrm{OH}, \mathrm{R}^{3}=\beta-\mathrm{OH}$

$76 \mathrm{R}^{1}=\mathrm{A}_{2}, \mathrm{R}^{2}=\beta-\mathrm{OH}, \mathrm{R}^{3}=\beta-\mathrm{OH}$

$77 \mathrm{R}^{1}=\mathrm{A}_{1}, \mathrm{R}^{2}=\beta-\mathrm{OH}, \mathrm{R}^{3}=\mathrm{H}$<smiles></smiles>

$78 \mathrm{R}^{1}=\mathrm{A}_{1}, \mathrm{R}^{2}=\alpha-\mathrm{OH}, \mathrm{R}^{3}=\mathrm{H}$

$79 \mathrm{R}^{1}=\mathrm{A}_{2}, \mathrm{R}^{2}=\beta-\mathrm{OH}, \mathrm{R}^{3}=\mathrm{H}$

$80 \mathrm{R}^{1}=\mathrm{A}_{2}, \mathrm{R}^{2}=\alpha-\mathrm{OH}, \mathrm{R}^{3}=\mathrm{H}$

$81 \mathrm{R}^{1}=\mathrm{A}_{3}, \mathrm{R}^{2}=\alpha-\mathrm{OH}, \mathrm{R}^{3}=\mathrm{H}$

$82 \mathrm{R}^{1}=\mathrm{A}_{4}, \mathrm{R}^{2}=\alpha-\mathrm{OH}, \mathrm{R}^{3}=\mathrm{H}$

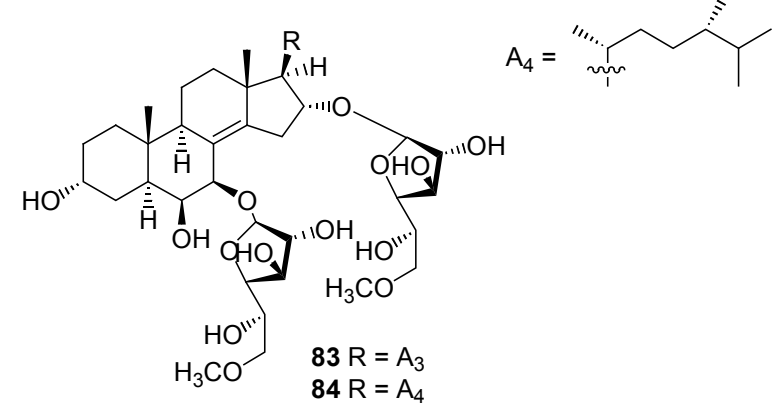

Ivanchina 等 ${ }^{[28]}$ 从远东海星 Henricia leviuscula. 的乙 醇提取液中分离得到九个甾体化合物 85 93, 其中化 合物 86 90 是文献中未报道过的新化合物, 进一步研 究发现：化合物 $85,87,90,91$ 和 93 均具有中等强度的溶 血活性. 对比这些化合物的结构和生理活性, 发现化合 物 86 与 85,87 相比较只是在支链结构上存在微小的变 化, 但是却出现了生理活性的显著差别.

Kicha 等 ${ }^{[29]}$ 从远东海星 Hippasteria kurilensis 的乙醇 提取液中分离出七种多羟基甾醇及甾体皇苷化合物 94 101, 研究发现化合物 94 98 以及化合物 100, 101 均有抑制海胆卵受精的作用. 通过比较化合物 99 和 100 的结构, 发现化合物 $\mathbf{1 0 0}$ 在 22,23-位存在着一个碳碳双 键, 正是受这个双键的影响使其具有抑制海胆卵受精的 活性, 而化合物 99 没有这个碳碳双键, 因此不具有此活 性.

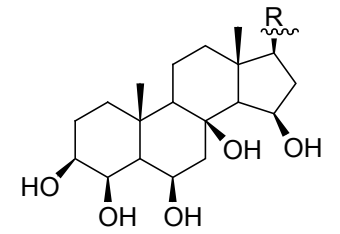

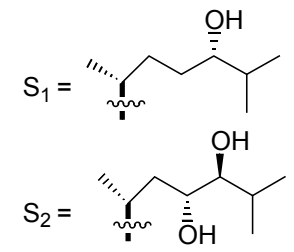

$85 R=S_{1} \quad 86 R=S_{2} \quad 87 R=S_{3}$<smiles>[R]C1C[C@H](O)[C@H]2[C@@H]3C[C@H](O)[C@@]4(O)C[C@@H](O)CC[C@]4(C)[C@H]3CC[C@]12C</smiles>

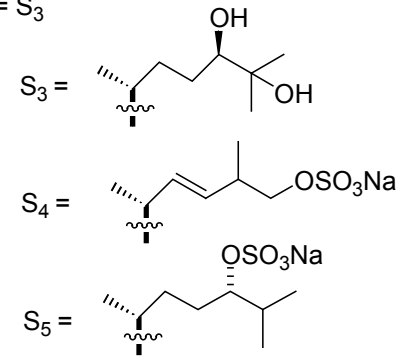

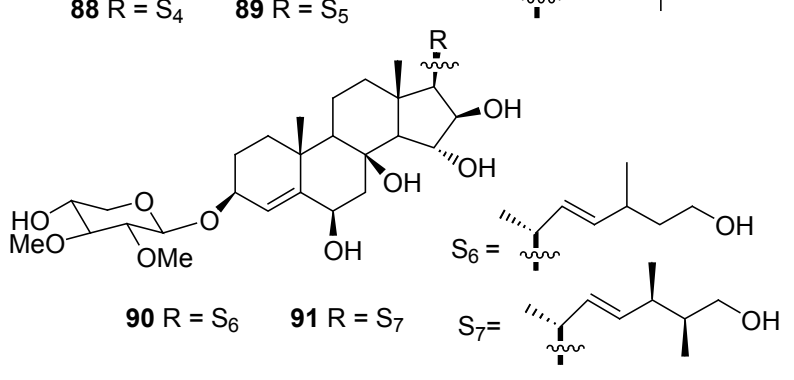

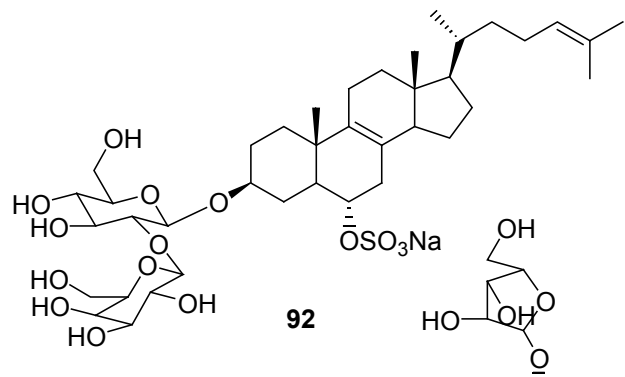<smiles>COC1OC[C@H](OC)C(OC(C)C)C[C@@H]1OC</smiles>

Levina 等 ${ }^{[30]}$ 从远东海星 Trofodiscus über 中分离得 到化合物 102 106, 其中化合物 102 104 都是未报道 过的新化合物，并进一步采用虾夷马粪海胆 Strongylocentrotus intermedius 的卵细胞对化合物进行活性研究, 分别测定了这些化合物的 $C_{\min }$ 值(使海胆未受精卵细胞 达静胞效应的最低溶度)、 $C_{\min }$ embr.值(第一次分裂阶段 终止海胆受精卵细胞分裂的最低溶度)、 $\mathrm{OC}_{50}$ 值(抑制海 胆精子细胞至一半的最低溶度)和 $\mathrm{IC}_{50}$ 值(抑制海胆受精 卵细胞受精至一半的溶度). 测试结果表明：化合物 102 106 在 $C_{\min }$ 为 $50 \mu \mathrm{g} / \mathrm{mL}$ 时, 对于海胆受精卵细胞 均没有活性; 化合物 103 在 $C_{\min }$ embr. 为 $50 \mu \mathrm{g} / \mathrm{mL}$ 时, 有很好的终止海胆第一次和第二次胚胎分裂的活性; 化 合物 102, 103, 105 和 106 具有较好的抑制海胆精子细胞 


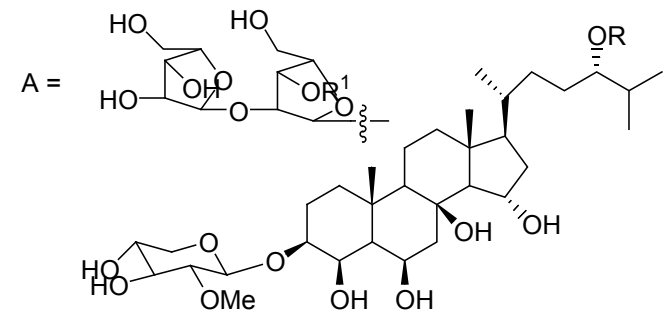

$94 \mathrm{R}=\mathrm{A}, \mathrm{R}^{1}=\mathrm{SO}_{3} \mathrm{Na}$ $95 \mathrm{R}=\mathrm{H}$

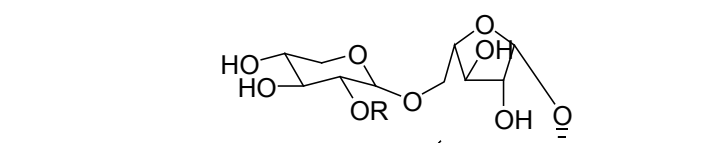

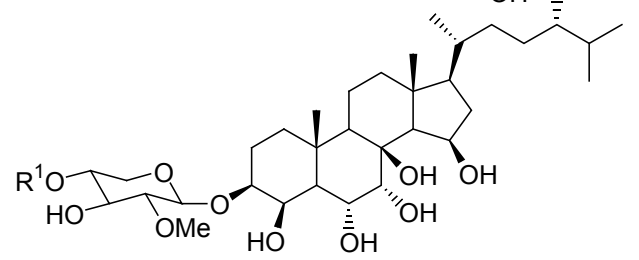

$96 \mathrm{R}=\mathrm{H}, \quad \mathrm{R}^{1}=\mathrm{Me}$

$97 \mathrm{R}=\mathrm{Me}, \mathrm{R}^{1}=\mathrm{H}$

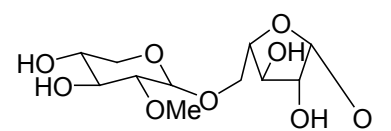

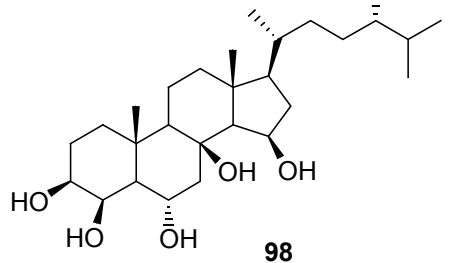

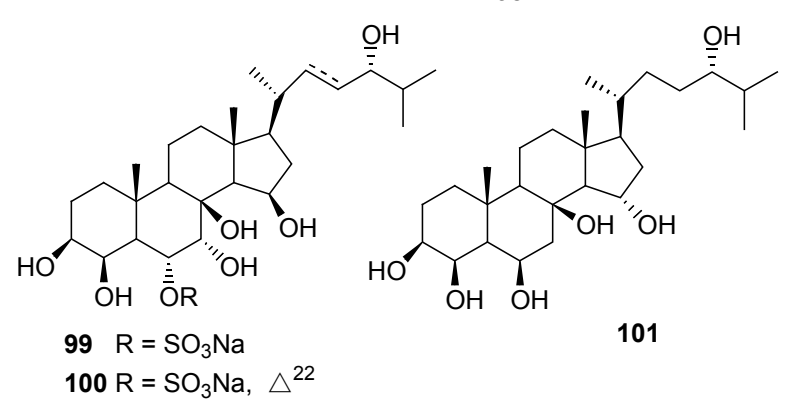

的活性, 其中化合物 102 对于海胆的精子细胞和胚胎细 胞均表现出很好的抑制活性 $\left(\mathrm{OC}_{50}\right.$ 值为 $12 \mu \mathrm{g} / \mathrm{mL}, \mathrm{IC}_{50}$ 值为 $6 \mu \mathrm{g} / \mathrm{mL}$ ), 并通过进一步研究发现含有磺酸基的多 差基甾醇对于抑制海胆卵细胞受精的活性最好.

\section{6 结语}

海洋丰富的生态资源环境已经成为人们拓展天然 药用资源的新空间, 多羟基甾醇就是其中一类具有显著 生理活性的海洋天然产物, 它们往往具有抗肿瘤、抗菌、 抗病毒、抗炎等生理活性, 具有很好的新药发展前景. 而且, 我们不难发现, 无论是在甾核环上还是在支链上, 结构上的微小差别都能够引起化合物在生理活性上的

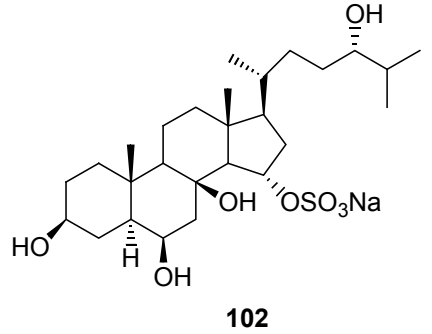

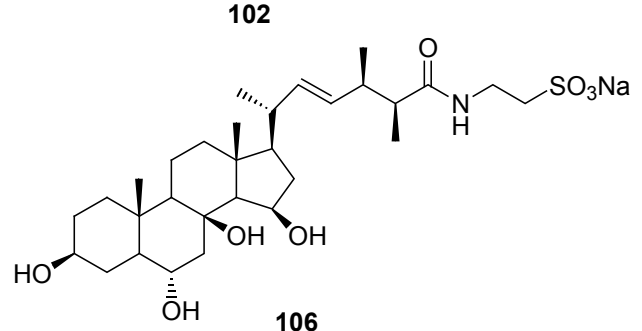

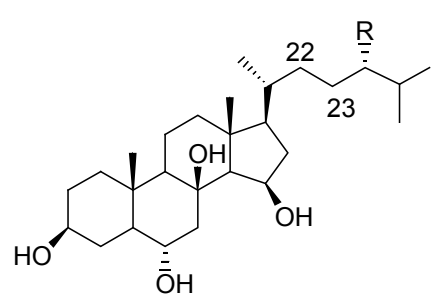

$103 \mathrm{R}=\mathrm{Xyl} 3 \mathrm{Me}$

$104 \mathrm{R}=\mathrm{Xyl} 3 \mathrm{Me}, 22(23)$-dehydro $105 \mathrm{R}=\mathrm{OH}$

显著差别. 因此, 以这些化合物作为药物研究的先导化 合物, 进一步进行结构改造和构效关系研究, 相信会有 越来越多结构新颖、生理活性显著的多羟基甾醇化合物 得到开发与利用, 为人类的健康做出贡献.

\section{References}

[1] Guo, Y.-W. Chin. J. Nat. 2009, 31(1), 27 (in Chinese). (郭跃伟，自然杂志, 2009, 31(1), 27.)

[2] Wang, F.-Z.; Fang, Y.-C.; Zhang, M.; Lin, A.-Q.; Zhu, T.-J.; Gu, Q.-Q.; Zhu, W.-M. Steroids 2008, 73(1), 19.

[3] Nguyen, H. T.; Chau, V. M.; Tran, T. H.; Phan, V. K.; Hoang, T. H.; Nguyen, T. D.; Nguyen, X. N.; Bui, H. T.; Hyun, J.-H.; Kang, H.-K.; Kim, Y. H. Bioorg. Med. Chem. Lett. 2009, 19(16), 4584.

[4] Marino, S. D.; Ummarino, R.; D'Auria, M. V.; Chini, M. G.; Bifulco, G.; D'Amore, C.; Renga, B.; Mencarelli, A.; Petek, S.; Fiorucci, S.; Zampella, A. Steroids 2012, 77(5), 484.

[5] Shin, K.; Chin, J.; Hahn, D.; Lee, J.; Hwang, H.; Won, D. H.; Ham, J.; Choi, H.; Kang, E.; Kim, H.; Ju, M. K.; Nam, S. J.; Kang, H. Steroids 2012, 77(5), 355.

[6] Su, J.-H.; Lo, C.-L.; Lu, Y.; Wen, Z.-H.; Huang, C.-Y.; Dai, C.-F.; Sheu, J.-H. Bull. Chem. Soc. Jpn. 2008, 81(12), 1616.

[7] Chao, C.-H.; Wen, Z.-H.; Chen, I.-M.; Su, J.-H.; Huang, H.-C.; Chiang, M. Y.; Sheu, J.-H. Tetrahedron 2008, 64(16), 3554.

[8] Chao, C.-H.; Wen, Z.-H.; Su, J.-H.; Chen, I.-M.; Huang, H.-C.; Dai, C.-F.; Sheu, J.-H. Steroids 2008, 73(14), 1353.

[9] Wang, S.-K.; Dai, C.-F.; Duh, C.-Y. J. Nat. Prod. 2006, 69(1), 103.

[10] Tran, H. Q.; Tran, T. H.; Chau, V. M.; Phan, V. K.; Huang, T. H.; Nguyen, T. T. N.; Nguyen, X. N.; Nguyen, H. T.; Nguyen, P. T.; Dinh, T. T. T.; Song, S. B.; Boo, H.-J.; Kang, H.-K.; Kim, Y. H. Bioorg. Med. Chem. Lett. 2011, $21(10), 2845$.

[11] Simmons, K.; Kaufmann, K.; Garcia, R.; Schwar, G.; Huch, V.; 
Muller, R. Bioorg. Med. Chem. 2011, $19(22), 6570$.

[12] Zhou, X.-F.; Lu, Y.-N.; Lin, X.-P.; Yang, B.; Yang, X.-W.; Liu, Y.-H. Chem. Phys. Lipids 2011, 164(7), 703.

[13] Ahmed, A. F.; Tai, S.-H.; Wu,Y.-C.; Sheu, J.-H. Steroids 2007, $72(4), 368$.

[14] Cheng, S.-Y.; Dai, C.-F.; Duh, C.-Y. Steroids 2007, 72(8), 653.

[15] Rao, T. S. P.; Sarma, N. S.; Murthy, Y. L. N.; Kantamreddi, V. S. S. N.; Wright, C. W.; Parameswaran, P. S. Tetrahedron Lett. 2010, 51(27), 3583.

[16] Sepe, V.; Ummarino, R.; D'Auria, M. V.; Chini, M. G.; Bifulco, G.; Renga, B.; D'Amore, C.; Debitus, C.; Fiorucci, S.; Zampella, A. J. Med. Chem. 2012, 55(1), 84.

[17] Huang, Y.-C.; Wen, Z.-H.; Wang, S.-K.; Hsu, C.-H.; Duh, C.-Y. Steroids 2008, 73(14), 1181.

[18] Sauleau, P.; Bourguet-Kondracki, M.-L. Steroids 2005, 70(14), 954.

[19] Dai, J.-Q.; Sorribas, A.; Yoshida, W. Y.; Kelly, M.; Williams, P. G. J. Nat. Prod. 2010, 73(9), 1597.

[20] Mitome, H.; Shirato, N.; Hoshino, A.; Miyaoka, H.; Yamada, Y.; Soest, R. W. M. V. Steroids 2005, 70(1), 63.

[21] Zhang, F.-Y.; Wang, N.; Geng, H.-W.; Liao, X.-J.; Xu, S.-H. Chin. J. Org. Chem. 2010, 30(11), 1745 (in Chinese).

(张福勇, 王楠, 耿华伟, 廖小建, 徐石海, 有机化学, 2010, 30(11), 1745.)

[22] Liao, X.-J.; Xu, S.-H.; Lin, H. Chin. J. Org. Chem. 2010, 30(5), 749 (in Chinese)

(廖小建, 徐石海, 林慧, 有机化学, 2010, 30(5), 749.)

[23] Zhang, G.-W.; Zhang, K.; Li, Y.-B.; Zeng, L.-M.;Su, J.-Y. Acta Sci. Nat. Univ. Sunyatseni 2007, 46(3), 68 (in Chinese).

(张广文, 张坤, 栗原博, 曾陇梅, 苏镜娱, 中山大学学报 (自然 科学版), 2007, 46(3), 68.)

[24] Zhang, C.-X.; Yan, S.-J.; Zhang, G.-W.; Su, J.-Y.; Zeng, L.-M. Chem. J. Chin. Univ. 2007, 28(4), 686 (in Chinese).

(张翠仙, 阎素君, 张广文, 苏镜娱, 曾陇梅, 高等学校化学学 报, 2007, 28(4), 686.)

[25] Chang, C.-H.; Wen, Z.-H.; Wang, S.-K.; Duh, C.-Y. Steroids 2008, 73(5), 562

[26] Wang, W.-H.; Jang, H.-J.; Hong, J.-K.; Lee, C.-O.; Bae, S.-J.; Shin, S.; Jung, J. H. Arch. Pharm. Res. 2005, 28(3), 285.

[27] Ma, N.; Tang, H.-F.; Qiu, F.; Lin, H.-W.; Tian, X.-R.; Yao, M.-N. J. Nat. Prod. 2010, 73(4), 590.

[28] Ivanchina, N. V.; Kicha, A. A.; Kalinovsky, A. I.; Dmitrenok, P. S.; Dmitrenok, A. S.; Chaikina, E. L.; Stonik, V. A.; Gavagnin, M.; Cimino, G. J. Nat. Prod. 2006, 69(2), 224.

[29] Kicha, A. A.; Ivanchina, N. V.; Kalinovsky, A. I.; Dmitrenok, P. S.; Agafonova, I. G.; Stonik,V. A. J. Nat. Prod. 2008, 71(5), 793.

[30] Levina, E. V.; Kalinovsky, A. I.; Andriyashchenko, P. V.; Menzorova, N. I.; Dmitrenok, P. S. Russ. J. Bioorg. Chem. 2007, 33(3), 334. 